\title{
THE TWO CONFORMAL INVARIANTS OF FIFTH ORDER*
}

\author{
BY \\ EDWARD KASNER
}

In the problem of classifying curvilinear angles in the plane with respect to the group of conformal transformations, the simplest invariant (other than angular magnitude $\theta$ ) which occurs is found in the case of a horn angle of first order contact (formula (1)). This is a differential invariant of third order, since it involves third derivatives of the two curves composing the angle. There are no invariants of fourth order (nor of any even order), but there are known to be just two of fifth order, arising in the cases of the horn angle of second order contact and the general right angle, respectively. $\dagger$ The main object of this paper is to determine the two invariants of fifth order explicitly. The results appear in formulas (15) and (23) below. $\ddagger$ It is shown elsewhere $\S$ how the latter of these expressions can be derived from the former through conformal symmetry. As a corollary we obtain in $\S 3$ the invariant of lowest order of a single curve under the inversion group, first found by G. W. Mullins. Generalizations to the case of angles drawn on a curved surface have been given by G. Comenetz.\|

I wish to acknowledge the valuable assistance of Miss A. Vassell and J. De Cicco in calculating the invariants, and of G. Comenetz in connection with $\$ 3$ and the use of oriented curves.

1. Horn angle of second order. We use the term curvilinear angle for the figure formed by an ordered pair of oriented, analytic curve-elements having a common point $O$, the vertex of the angle.

The symbol $\gamma$ is employed for the curvature of a curve, $\gamma^{\prime}$ for the derivative $d \gamma / d s$ with respect to arc-length $s, \gamma^{\prime \prime}$ for the second derivative $d^{2} \gamma / d s^{2}$, and so on. Given a curvilinear angle, the symbols, $\gamma_{1}, \gamma_{1}^{\prime}, \gamma_{1}^{\prime \prime}, \cdots$ denote the curvature and its successive arc-length derivatives for the first side of the angle, evaluated at the vertex $O$; and $\gamma_{2}, \gamma_{2}^{\prime}, \gamma_{2}^{\prime \prime}, \ldots$ are the corresponding

* Presented to the Society, September 10, 1937; received by the editors June 11, 1937. Abstract in Science, vol. 82 (1935), p. 622.

$\dagger$ E. Kasner, Conformal geometry, Proceedings of the Fifth International Congress of Mathematicians, Cambridge, 1912, vol. 2, p. 81.

$\ddagger$ The next simplest invariants, of seventh order, would be encountered in dealing with the horn angle of third order contact and the general $60^{\circ}$ angle. The latter is being studied by S. H. Gould and O. P. Doria.

$\S$ See E. Kasner, Conformal symmetry (Schwarzian reflexion), Annals of Mathematics, vol. 38 (1937), pp. 873-879.

|| Bulletin of the American Mathematical Society, abstract 42-11-399. 
quantities for the second side of the angle. The conformal invariants of curvilinear angles given below are all stated in terms of the metric invariants $\gamma_{1}, \gamma_{2}, \cdots, \gamma_{1}^{\prime \prime \prime}, \gamma_{2}^{\prime \prime \prime}$.

A curvilinear angle is called a horn angle if the two curves coincide in direction at the vertex, and is described as being of $k$ th order contact $(k=1,2, \cdots)$ if the order of contact of the curves at the point of tangency is exactly $k$. A horn angle of $k$ th order contact is called an $H_{k+1}$. The conformal invariant of an $\mathrm{H}_{2}$, as the author has shown, is given by*

$$
I_{12}=\frac{\gamma_{2}^{\prime}-\gamma_{1}^{\prime}}{\left(\gamma_{2}-\gamma_{1}\right)^{2}}
$$

To derive the analogous invariant for an $\mathrm{H}_{3}$ we proceed as follows.

Suppose that an $H_{3}$ lying in the $(x, y)$-plane is transformed conformally into an $H_{3}$ lying in the $(X, Y)$-plane. We may assume that the coordinates are so chosen in each plane that the vertex of the angle is the origin and the initial tangent direction is that of the positive axis of abscissas. Then in the $(x, y)$ plane the first side of the angle is given by

$$
y=a_{2} x^{2}+a_{3} x^{3}+\cdots
$$

and the second side by

$$
y=a_{2}^{\prime} x^{2}+a_{3}^{\prime} x^{3}+\cdots .
$$

Here $a_{2}=a_{2}^{\prime}$ and $a_{3} \neq a_{3}^{\prime}$, because the contact is of second order (exactly). In the $(X, Y)$-plane the first and second sides of the transformed angle are given respectively by

$$
Y=A_{2} X^{2}+A_{3} X^{3}+\cdots
$$

and

$$
Y=A_{2}^{\prime} X^{2}+A_{3}^{\prime} X^{3}+\cdots,
$$

with $A_{2}=A_{2}^{\prime}$ and $A_{3} \neq A_{3}^{\prime}$. (All the curves are oriented to the right.)

If we set $z=x+i y$ and $w=X+i Y$, the conformal transformation can be represented in some neighborhood of the origin by

$$
w=c_{1} z+c_{2} z^{2}+\cdots,
$$

where the coefficients $c_{n}$ are complex $\left(c_{n}=\alpha_{n}+i \beta_{n}\right)$, and $c_{1}$ is not zero. As the initial direction of the positive axis of abscissas remains fixed, $c_{1}$ is necessarily real and positive; $c_{1}=\alpha_{1}>0$. To simplify the calculations we shall as-

* See Cambridge paper, and papers by Kasner and Comenetz, Proceedings of the National Academy of Sciences and the American Mathematical Monthly, 1936-1938. 
sume at first that $c_{1}=1$, so that instead of (4) we have

$$
w=z+c_{2} z^{2}+c_{3} z^{3}+\cdots \text {. }
$$

It will be easy to allow for the more general transformation (4) later.

Separating $\left(4^{\prime}\right)$ into real and imaginary parts, we obtain the equations of the transformation in the real form

$$
\begin{aligned}
& X=x+\alpha_{2}\left(x^{2}-y^{2}\right)-2 \beta_{2} x y+\alpha_{3}\left(x^{3}-3 x y^{2}\right)+\cdots, \\
& Y=y+2 \alpha_{2} x y+\beta_{2}\left(x^{2}-y^{2}\right)+\alpha_{3}\left(3 x^{2} y-y^{3}\right)+\cdots .
\end{aligned}
$$

Substituting (5) into (3) and eliminating $y$ by means of (2), we derive an identity in $x$. If we equate the coefficients of $x^{2}, \cdots, x^{5}$, respectively, and solve for $A_{2}, \cdots, A_{5}$, after some computation we find the following relations:

(61) $A_{2}=a_{2}+\beta_{2}$,

(6) $\quad A_{3}=a_{3}+\beta_{3}-2 \alpha_{2} \beta_{2}$,

(6) $A_{4}=a_{4}-\alpha_{2} a_{3}+3 \beta_{2} a_{2}^{2}+\left(\alpha_{3}-\alpha_{2}^{2}+4 \beta_{2}^{2}\right) a_{2}+\beta_{4}-2 \alpha_{3} \beta_{2}$

$$
-3 \alpha_{2} \beta_{3}+5 \alpha_{2}^{2} \beta_{2} \text {, }
$$

(6) $\quad A_{5}=a_{5}-2 \alpha_{2} a_{4}+8 \beta_{2} a_{2} a_{3}+\left(\alpha_{2}{ }^{2}+4{\beta_{2}}^{2}\right) a_{3}+2 \alpha_{2} a_{2}{ }^{3}$

$$
\begin{aligned}
& +\left(3 \beta_{3}-6 \alpha_{2} \beta_{2}\right) a_{2}^{2}+\left(2 \alpha_{4}-6 \alpha_{2} \alpha_{3}+4 \alpha_{2}{ }^{3}-24 \alpha_{2} \beta_{2}{ }^{2}+12 \beta_{2} \beta_{3}\right) a_{2} \\
& +\beta_{5}-2 \beta_{2} \alpha_{4}-4 \alpha_{2} \beta_{4}-3 \alpha_{3} \beta_{3}+12 \alpha_{2} \beta_{2} \alpha_{3}+9 \alpha_{2}{ }^{2} \beta_{3}-14 \alpha_{2}{ }^{3} \beta_{2} .
\end{aligned}
$$

Of course the corresponding relations $\left(\sigma_{1}^{\prime}\right)-\left(\sigma_{4}^{\prime}\right)$ for the second side of the angle are obtained from these by replacing $a_{2}, \cdots, a_{5}, A_{2}, \cdots, A_{5}$ by $a_{2}^{\prime}, \cdots, a_{5}^{\prime}, A_{2}^{\prime}, \cdots, A_{5}^{\prime}$, respectively.

In order to find invariants we must eliminate the coefficients $\alpha_{n}, \beta_{n}$ of the transformation. To eliminate $\beta_{5}$ we subtract $\left(6_{4}\right)$ from $\left(6_{4}^{\prime}\right)$, recalling that $a_{2}=a_{2}^{\prime}$. The result is

$$
\Delta A_{5}=\Delta a_{5}-2 \alpha_{2} \Delta a_{4}+8 \beta_{2} a_{2} \Delta a_{3}+\left(\alpha_{2}^{2}+4 \beta_{2}^{2}\right) \Delta a_{3},
$$

where $\Delta A_{j} \equiv A_{j}^{\prime}-A_{j}, \Delta a_{j} \equiv a_{j}^{\prime}-a_{i}(j=3,4,5)$. Similarly we eliminate $\beta_{4}$ and $\beta_{3}$ by forming the differences

$$
\begin{aligned}
& \Delta A_{4}=\Delta a_{4}-\alpha_{2} \Delta a_{3}, \\
& \Delta A_{3}=\Delta a_{3} .
\end{aligned}
$$

Finally $\alpha_{2}$ and $\beta_{2}$ are eliminated by substituting from $\left(6_{1}\right)$ and (8) into (7). Rearranging and using (9), we find that

$$
\Delta A_{3} \Delta A_{5}-\left(\Delta A_{4}\right)^{2}-4 A_{2}{ }^{2}\left(\Delta A_{3}\right)^{2}=\Delta a_{3} \Delta a_{5}-\left(\Delta a_{4}\right)^{2}-4 a_{2}{ }^{2}\left(\Delta a_{3}\right)^{2} .
$$

Equations (9) and (10) exhibit two quantities which are invariant under 
the special transformation $\left(4^{\prime}\right)$. If we follow $\left(4^{\prime}\right)$ by a magnification

$$
w=\alpha_{1} z,
$$

$\alpha_{1}>0$,

the resultant is a general conformal transformation of the type (4). It is easy to see that the effect of (11) on a curve (2) is to divide the coefficient $a_{n}$ by $\alpha_{1}{ }^{n-1}$. Consequently (9) and (10) still hold under the general transformation (4) provided we insert the factors $1 / \alpha_{1}{ }^{2}$ and $1 / \alpha_{1}{ }^{6}$ in the right members of (9) and (10), respectively.

The coefficients of the curve (2) are expressed in terms of the curvature and its arc-length derivatives at the origin by the standard formulas

$$
\begin{aligned}
2 a_{2} & =\gamma_{1}, \\
6 a_{3} & =\gamma_{1}^{\prime}, \\
24 a_{4} & =\gamma_{1}^{\prime \prime}+3 \gamma_{1}^{3}, \\
120 a_{5} & =\gamma_{1}^{\prime \prime \prime}+19 \gamma_{1}^{2} \gamma_{1}^{\prime} .
\end{aligned}
$$

Using these formulas and the similar ones for the curve $\left(2^{\prime}\right)$, we find that the right members of (9) and (10) can be written, except for numerical factors, as

$$
\gamma_{2}^{\prime}-\gamma_{1}^{\prime}
$$

and

$$
4\left(\gamma_{2}^{\prime}-\gamma_{1}^{\prime}\right)\left(\gamma_{2}^{\prime \prime \prime}-\gamma_{1}^{\prime \prime \prime}\right)-5\left(\gamma_{2}^{\prime \prime}-\gamma_{1}^{\prime \prime}\right)^{2}-4 \gamma^{2}\left(\gamma_{2}^{\prime}-\gamma_{1}^{\prime}\right)^{2},
$$

respectively, where $\gamma \equiv \gamma_{1}=\gamma_{2}$. Now the Jacobian at $O$ of the transformation (4) is $\alpha_{1}{ }^{2}$. Consequently we can state this theorem:

THEOREM I. The quantities (13) and (14) are relative invariants of a horn angle of second order contact under conformal transformation. They are of index 1 and 3 respectively.

In particular the sign of (13) is invariant, since the Jacobian is positive. The fundamental theorem is as follows:

Theorem II. The quantity

$$
\frac{4\left(\gamma_{2}^{\prime}-\gamma_{1}^{\prime}\right)\left(\gamma_{2}{ }^{\prime \prime \prime}-\gamma_{1}{ }^{\prime \prime \prime}\right)-5\left(\gamma_{2}{ }^{\prime \prime}-\gamma_{1}{ }^{\prime \prime}\right)^{2}-4 \gamma^{2}\left(\gamma_{2}^{\prime}-\gamma_{1}^{\prime}\right)^{2}}{\left(\gamma_{2}^{\prime}-\gamma_{1}^{\prime}\right)^{3}}
$$

and the sign of $\gamma_{2}^{\prime}-\gamma_{1}^{\prime}$ are invariants of a horn angle of second order contact under conformal transformation. Conversely, if two such angles agree in the value of (15) and the sign of (13) they can be made to coincide by a conformal transformation (at least to any finite order of contact).*

* A similar theorem holds for an $H_{2}$, the value of (1) and the sign of $\gamma_{2}-\gamma_{1}$ being the corresponding invariants. 
The first statement follows from Theorem I. For the proof of the second (converse) statement we refer to the Cambridge paper, where the method of conformal symmetry is applied.

2. General curvilinear right angle. A curvilinear right angle is said to be a "general" right angle provided $\gamma_{2}^{\prime}+\gamma_{1}^{\prime} \neq 0$.*

Let the first side of the angle be given by (2), and the second side by

$$
x=b_{2} y^{2}+b_{3} y^{3}+\cdots
$$

(oriented upwards). Let the transformed first side be given by (3) and the second side by

$$
X=B_{2} Y^{2}+B_{3} Y^{3}+\cdots .
$$

Again we begin with a transformation of the special type $\left(4^{\prime}\right)$.

In deducing the equations analogous to $\left(6_{1}\right)-\left(6_{4}\right)$ for the second side of the angle, we can avoid a new calculation if we observe that $\left(4^{\prime}\right)$ can be written as

$Y+i X=(y+i x)+\frac{1}{i}\left(\alpha_{2}-i \beta_{2}\right)(y+i x)^{2}+\frac{1}{i^{2}}\left(\alpha_{3}-i \beta_{3}\right)(y+i x)^{3}+\cdots$.

This shows that if we interchange $X$ and $Y, x$ and $y, A$ and $B$, and $a$ and $b$, and furthermore replace $\alpha_{2}, \beta_{2}, \alpha_{3}, \beta_{3}, \alpha_{4}, \beta_{4}, \alpha_{5}, \beta_{5}$, by $-\beta_{2},-\alpha_{2},-\alpha_{3}, \beta_{3}$, $\beta_{4}, \alpha_{4}, \alpha_{5},-\beta_{5}$, respectively, then the calculation that was made for $\left(6_{1}\right)-\left(6_{4}\right)$ will turn into the calculation which we now require. Consequently to obtain the new relations we need only make the above interchanges and replacements in equations $\left(6_{1}\right)-\left(6_{4}\right)$. The result is

(182) $\quad B_{3}=b_{3}+\beta_{3}-2 \alpha_{2} \beta_{2}$,

$\left(18_{3}\right) \quad B_{4}=b_{4}+\beta_{2} b_{3}-3 \alpha_{2} b_{2}^{2}+\left(-\alpha_{3}+4 \alpha_{2}^{2}-\beta_{2}^{2}\right) b_{2}$

$$
+\alpha_{4}-2 \alpha_{2} \alpha_{3}+3 \beta_{2} \beta_{3}-5 \alpha_{2} \beta_{2}{ }^{2} \text {, }
$$

$$
\begin{aligned}
B_{5}=b_{5} & +2 \beta_{2} b_{4}-8 \alpha_{2} b_{2} b_{3}+\left(4 \alpha_{2}{ }^{2}+\beta_{2}{ }^{2}\right) b_{3}-2 \beta_{2} b_{2}{ }^{3}+\left(3 \beta_{3}-6 \alpha_{2} \beta_{2}\right) b_{2}{ }^{2} \\
& +\left(2 \beta_{4}-6 \beta_{2} \alpha_{3}-12 \alpha_{2} \beta_{3}+24 \alpha_{2}{ }^{2} \beta_{2}-4 \beta_{2}{ }^{3}\right) b_{2}-\beta_{5}+4 \beta_{2} \alpha_{4} \\
& +2 \alpha_{2} \beta_{4}+3 \alpha_{3} \beta_{3}-12 \alpha_{2} \beta_{2} \alpha_{3}+9 \beta_{2}{ }^{2} \beta_{3}-14 \alpha_{2} \beta_{2}{ }^{3} .
\end{aligned}
$$

To eliminate $\beta_{5}$ we add $\left(6_{4}\right)$ and $\left(18_{4}\right)$. Then we subtract $2 A_{2} B_{4}+2 B_{2} A_{4}$ from the sum to eliminate $\alpha_{4}$ and $\beta_{4}$. Next the subtraction of $3\left(A_{2}{ }^{2}+B_{2}{ }^{2}\right)\left(A_{3}+B_{3}\right) / 2$

* An equivalent definition is that the curve obtained on reflecting the first side of the angle in the second side (by conformal symmetry or Schwarzian reflection) and then reversing orientation, forms an $\mathrm{H}_{3}$ with the first side. 
removes $\beta_{3}$. Finally we add $-5\left(A_{2}{ }^{2}-B_{2}{ }^{2}\right)\left(A_{3}-B_{3}\right) / 2+2 B_{2} A_{2}{ }^{3}+2 A_{2} B_{2}{ }^{3}$. In this manner we obtain two expressions which are invariant under the special transformation $\left(4^{\prime}\right)$, namely,

$$
b_{3}-a_{3}
$$

(20) $b_{5}+a_{5}-2 a_{2} b_{4}-2 b_{2} a_{4}-4 b_{2}{ }^{2} b_{3}+a_{2}{ }^{2} b_{3}+b_{2}{ }^{2} a_{3}-4 a_{2}{ }^{2} a_{3}+2 a_{2} b_{2}{ }^{3}+2 b_{2} a_{2}{ }^{3}$.

Under the general transformation (4), (19) would be multiplied by $1 / \alpha_{1}{ }^{2}$ and (20) by $1 / \alpha_{1}{ }^{4}$.

In introducing curvatures for the vertical curve (16) we must be careful to note that the proper formulas are the negatives of $\left(12_{1}\right)-\left(12_{4}\right)$; thus $2 b_{2}=-\gamma_{2}$, $6 b_{3}=-\gamma_{2}^{\prime}$, and so on. Substituting in (19) and (20) we find

$$
\begin{gathered}
\gamma_{2}^{\prime}+\gamma_{1}^{\prime}, \\
\left(\gamma_{2}^{\prime \prime \prime}-\gamma_{1}^{\prime \prime \prime}\right)-5\left(\gamma_{1} \gamma_{2}^{\prime \prime}+\gamma_{2} \gamma_{1}^{\prime \prime}\right)-\gamma_{2}^{2} \gamma_{2}^{\prime}+5 \gamma_{1}^{2} \gamma_{2}^{\prime}-5 \gamma_{2}^{2} \gamma_{1}^{\prime}+\gamma_{1}^{2} \gamma_{1}^{\prime} .
\end{gathered}
$$

THEOREM III. The quantities (21) and (22) are relative conformal invariants of any curvilinear right angle. They are of index 1 and 2, respectively.

THEOREM IV. The quantity

$$
\frac{\left(\gamma_{2}{ }^{\prime \prime \prime}-\gamma_{1}{ }^{\prime \prime \prime}\right)-5\left(\gamma_{1} \gamma_{2}^{\prime \prime}+\gamma_{2} \gamma_{1}{ }^{\prime \prime}\right)-\gamma_{2}{ }^{2} \gamma_{2}^{\prime}+5 \gamma_{1}{ }^{2} \gamma_{2}^{\prime}-5 \gamma_{2}{ }^{2} \gamma_{1}^{\prime}+\gamma_{1}{ }^{2} \gamma_{1}^{\prime}}{\left(\gamma_{2}^{\prime}+\gamma_{1}^{\prime}\right)^{2}}
$$

and the sign of $\gamma_{2}^{\prime}+\gamma_{1}^{\prime}$ are conformal invariants of a general right angle. Conversely, if two such angles agree in the value of (23) and the sign of (21), they can be made to coincide by a conformal transformation (at least to any finite order of contact).*

The proof of the converse portion of the theorem is given in the Cambridge paper.

3. Inversive invarients. An invariant under the conformal group is of course also an invariant under the inversion group, defined by

$$
w=\frac{a z+b}{c z+d}
$$

* If the orientation of the second side is reversed, a general right angle becomes a general $270^{\circ}$ angle. The effect of reversing orientation is to change the signs of $\gamma_{2}, \gamma_{2}^{\prime \prime}, \gamma_{2}{ }^{\text {iv }}, \cdots$ but to leave $\gamma_{2}^{\prime}, \gamma_{2}^{\prime \prime \prime}, \cdots$ unaltered. It follows that if we replace $\gamma_{2}$ by $-\gamma_{2}$ and $\gamma_{2}^{\prime \prime}$ by $-\gamma_{2}^{\prime \prime},(23)$ becomes the invariant of a general $270^{\circ}$ angle. The same replacements convert (1) and (15) into the invariants of (curvilinear) straight angles of first and second order contact respectively.

All the invariants mentioned in this paper apply also to angles whose sides merely possess fifth derivatives (for (1), third derivatives) at the vertex. This follows from the fact that such an angle can be approximated to fifth (or third) order by an analytic angle, and the fact that a conformal transformation preserves contact. The converses in Theorems II and IV are true only for analytic angles, however. 
since the latter is a subgroup of the former.

Consider the horn angle of second order contact formed by a curve and its osculating circle. The value of the invariant (15) in this case is evidently

$$
\frac{-4 \gamma^{\prime} \gamma^{\prime \prime \prime}+5 \gamma^{\prime \prime 2}+4 \gamma^{2} \gamma^{\prime 2}}{\gamma^{\prime 3}},
$$

where $\gamma, \gamma^{\prime}, \ldots$ represent curvature and its arc-length derivatives for the given curve. Now inversion preserves the circle of curvature. Consequently (24) is an invariant of a single curve under the inversion group. This was first proved by G. W. Mullins, who showed that (24) is in fact the inversive invariant of lowest order and gave all the higher invariants.*

The question may be raised, for what curves does the value of (1) as formed for the horn angle of first order contact between the curve and its tangent line, remain constant along the curve? (These horn angles at any two points of the curve would then be conformally equivalent, at least to any finite order of contact.) The condition is $-\gamma^{\prime} / \gamma^{2}=$ const., or $d \rho / d s=$ const. This is the intrinsic equation of the logarithmic spirals.

The analogous question for the horn angle of second order contact between a curve and its osculating circle can be solved by using a theorem of Mullins. In that case the curves are the logarithmic double spirals, which may be defined as the transforms by inversion of logarithmic spirals. $\dagger$

Trihornometry for general horn angles of second order contact will be discussed in later papers. For each order a new type of Finsler space must be introduced. The total geometry is non-archimedian.

* Differential Invariants under the Inversion Group, Columbia University Dissertation, 1917, p. 21. The $I_{5}$ there is identical with (24) above (with $\rho=1 / \gamma$ ).

$\dagger$ Or as the isogonal trajectories of a pencil of circles through two fixed points, or as the images of loxodromes on a sphere under stereographic projection from an arbitrary point of the sphere (G. Holzmüller, Ueber die logarithmische Abbildung und die aus ihr entspringenden orthogonalen Curvensysteme, Zeitschrift für Mathematik und Physik, vol. 16 (1871), p. 269).

Columbia University, NEW YORK, N. Y. 